\title{
A systematic review and meta-analysis of clinical outcomes of vitrectomy with or without intravitreal bevacizumab pretreatment for severe diabetic retinopathy
}

\author{
Li-Quan Zhao, Huang Zhu, Pei-Quan Zhao, Yi-Qian Hu
}

Department of Ophthalmology, Xinhua Hospital Affiliated to Shanghai Jiao Tong University School of Medicine, Shanghai, PR China

\section{Correspondence to}

Dr Huang Zhu, Department of Ophthalmology, Xinhua Hospital Affiliated to Shanghai Jiao Tong University School of Medicine, 1665 Kongjiang Road, Shanghai, 200092, PR China: zhuwjp@sh163.net

Accepted 21 November 2010 Published Online First 27 January 2011

\section{ABSTRACT}

Aims To examine possible benefits of intravitreal bevacizumab (IVB) pretreatment in vitrectomy for severe diabetic retinopathy.

Methods A comprehensive literature search was performed using the Cochrane Collaboration methodology to identify randomised controlled trials and comparative studies of vitrectomy with or without IVB pretreatment for severe or complicated diabetic retinopathy. Meta-analyses were performed for intraoperative (including intraoperative bleeding, endodiathermy, iatrogenic retinal tears and mean surgical time) and postoperative outcome parameters (including best-corrected visual acuity, recurrent vitreous haemorrhage, reabsorption time of blood and other complications).

Results Six randomised controlled trials and one comparative study were identified and used for comparing vitrectomy alone (142 eyes, control group) with vitrectomy with IVB pretreatment (139 eyes). The intraoperative findings showed that the incidence of intraoperative bleeding and frequency of endodiathermy were statistically significantly less in the IVB pretreatment group $(p<0.01)$ than in the vitrectomy alone group. The IVB pretreatment group took significantly less surgical time than the control group $(p=0.003)$. Postoperative results indicated that reabsorption time of blood was significantly shorter $(p=0.04)$, incidence of recurrent $\mathrm{VH}$ was almost significantly less $(p=0.05)$, and final best-corrected visual acuity was significantly better $(p=0.003)$ in the IVB group than in the control group. Other complications, including final retinal detachment, and reoperation, were statistically insignificant.

Conclusion IVB pretreatment in vitrectomy can achieve excellent clinical outcomes for severe diabetic retinopathy. It potentially facilitates surgeons' manoeuvres and reduces intra- and postoperative complications.

\section{INTRODUCTION}

Recently, numerous studies have reported clinical outcomes of intravitreal bevacizumab (IVB) as an adjunct to vitrectomy in the management of diabetic retinopathy. ${ }^{1-10}$ Bevacizumab can induce regression of retinal neovascularisation in patients with diabetes; therefore, it was suggested that a presurgical administration of IVB may reduce intraoperative bleeding during vitrectomy in proliferative diabetic retinopathy (PDR). ${ }^{1}{ }^{3-6}$ However, the presurgical administration of IVB remains controversial. Some studies reported that bevacizumab pretreatment for diabetic vitrectomy did not influence rates of postoperative vitreous haemorrhage or final visual acuity. 710

Although many surgeons perform IVB before vitrectomy in patients with diabetes, systematic or larger sample size studies demonstrating its benefits in facilitation of surgery and clinical outcomes are limited. Therefore, it is necessary to review in greater depth the available studies to understand the benefits of IVB pretreatment. In an attempt to detect benefits in safety and efficacy as the primary comparative criteria, we performed a system review and meta-analysis of existing RCTs and highquality comparative studies of vitrectomy with or without IVB pretreatment for the treatment of severe or complicated diabetic retinopathy.

\section{MATERIALS AND METHODS}

This meta-analysis was performed according to a predetermined protocol described previously. ${ }^{11} 12$

\section{Literature search}

Two reviewers independently searched the following electronic databases: PubMed, EMBASE and the Cochrance Controlled Trials Register up to 30 April 2010. For maximum sensitivity, we used free text and thesaurus terms including 'vitrectomy,' 'diabetic retinopathy' and 'bevacizumab.' Full articles were retrieved, when titles and/or abstracts met this objective. A manual cross-reference search of the bibliographies of relevant articles was conducted. All published studies comparing vitrectomy alone versus vitrectomy with IVB pretreatment for diabetic retinopathy were included, if they met the inclusion criteria. The search included RCTs and high-quality comparative studies. For an inclusion in the meta-analysis, the patients in the selected studies had to present with severe diabetic retinopathy and be older than 18 years of age. At least one or more clinical outcomes representing intraoperative and/or postoperative outcome parameters must be assessed and published. There was no language restriction on the publications.

Discordance about study inclusion between the two reviewers was resolved through discussion until $100 \%$ agreement was reached on the final interpretation of the data.

\section{Quality assessment of retrieved articles}

The selected studies were appraised by two reviewers, who independently assessed their quality 
using the methods recommended in the Cochrane Handbook for Systematic Reviews of Interventions. ${ }^{13}$ Methodological quality included allocation concealment, method of allocation to treatment, masking of outcome assessment and completeness of follow-up.

\section{Outcome measure}

The intraoperative outcome parameters included incidence of intraoperative bleeding, frequency of endodiathermy, iatrogenic retinal tears and mean surgical time. Postoperative outcome parameters included best-corrected visual acuity (BCVA), recurrent vitreous haemorrhage $(\mathrm{VH})$, reabsorption time of blood, early and late elevation of intraocular pressure (IOP), final retinal detachment (RD) and repeat vitrectomy.

\section{Data extraction and analysis}

The studies were tabulated and methodologically evaluated to assess homogeneity. Any heterogeneity between the studies would not be justified to pool the assessed outcomes. A customised data-extraction form, as described in the Cochrane Handbook for Systematic Reviews of Interventions, was used to record the duration of the trial, sample size, dropouts, the system and ocular baseline features, the type of surgery and differential treatment, the dosage and time of bevacizumab injection.

\section{Statistical analysis}

Quantitative data were analysed using the Cochrane Review Manager (RevMan) version 4.2 software. Summary estimates, including $95 \% \mathrm{CIs}$, were calculated. For continuous outcome data (eg, mean surgical time), means and standard deviations were used to calculate a weighted mean difference (WMD). For dichotomous outcomes (eg, proportions of intraoperative bleeding), the OR was calculated.

Statistical heterogeneity was tested using the $Q$ statistic of the $\chi^{2}$ value test and $\mathrm{I}^{2}$ test. Fixed-effects models were used, unless significant evidence of statistical heterogeneity or clinical diversity was found. For results showing significant heterogeneity $\left(\mathrm{I}^{2}>50 \%\right)$, a random-effects meta-analysis was performed by the DerSimonian-Laird method. Outcome measures were assessed on an intent-to-treat basis. A p value of $<0.05$ was considered statistically significant. A sensitivity analysis was performed by excluding the non-randomised study.

Publication bias was assessed by visually inspecting a funnel plot.

\section{RESULTS}

Six RCTs and one comparative study published between 2008 and 2010 met the inclusion criteria. ${ }^{1-69}$ In di Lauro's study, the group of IVB 7 days prior to vitrectomy was selected to the IVB group. ${ }^{9}$ The present meta-analysis involved 142 eyes treated with vitrectomy alone and 139 eyes receiving vitrectomy with IVB pretreatment. The selection of seven studies is summarised in tables 1,2. Each study revealed that there were no significant differences in preoperative demographic features and other factors that may have influenced surgery, such as previous panretinal photocoagulation.

\section{Intraoperative outcome parameters}

Five studies reported data for intraoperative bleeding. ${ }^{13569}$ The studies applied differential scales for the evaluation of intraoperative bleeding. Clinically significant intraoperative bleeding (moderate to severe) was included in this meta-analysis.

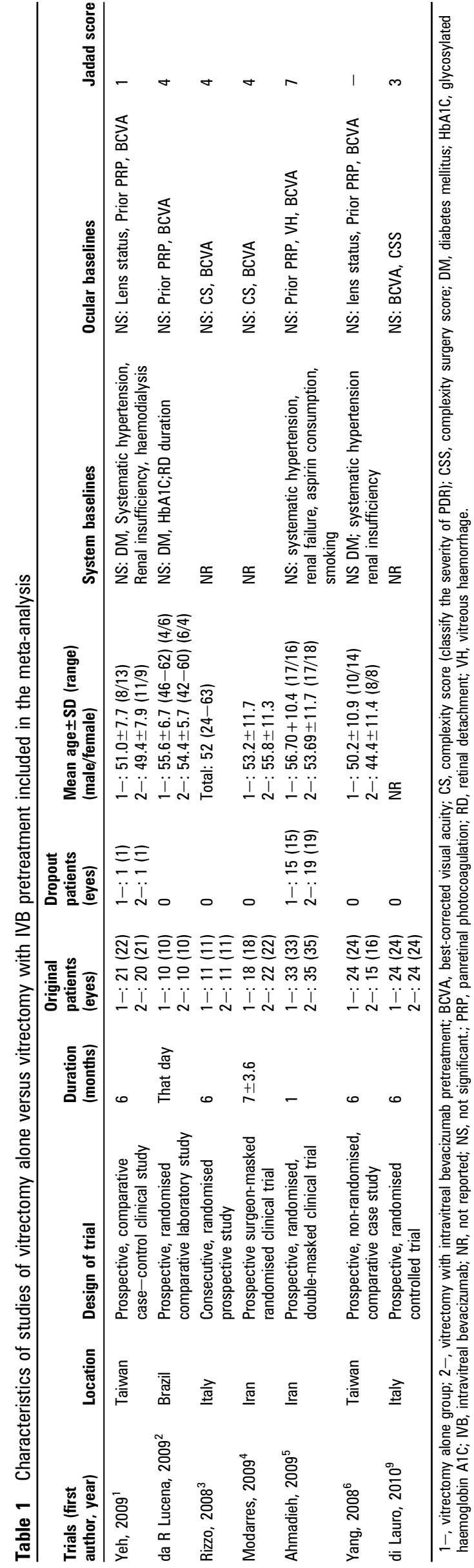




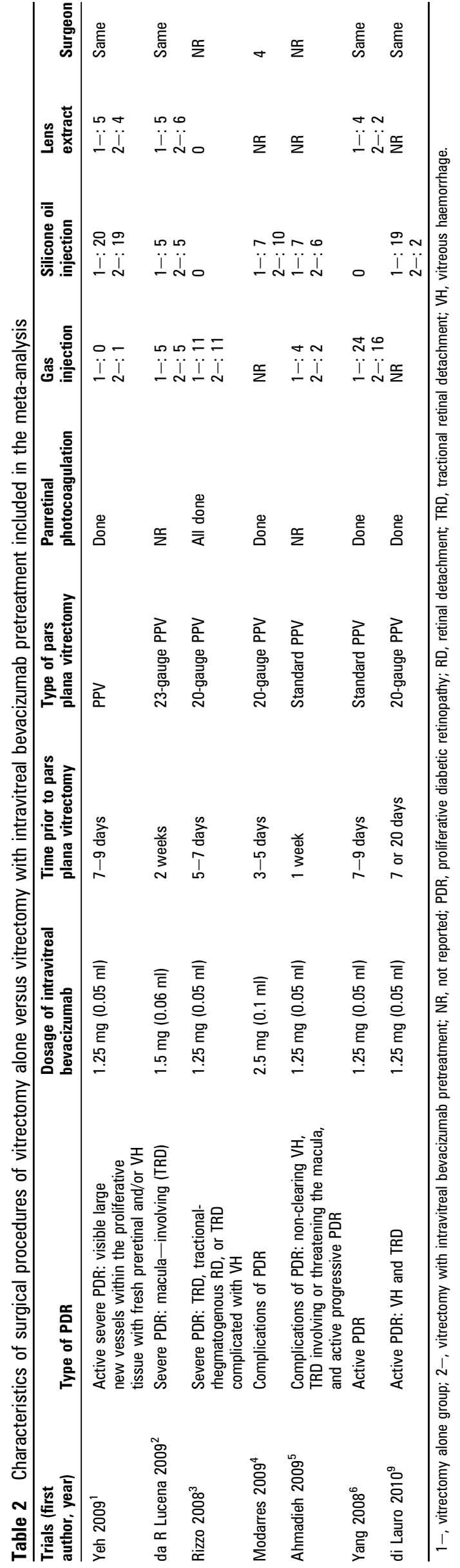

Analysis of these data showed that the incidence of intraoperative bleeding was statistically significantly less in the IVB pretreatment group than in the vitrectomy alone group (OR 8.85; $95 \%$ CI 2.08 to $37.61 ; p=0.003$ ) (figure $1 \mathrm{~A}$ ). A sensitivity analysis was performed to examine the effect of excluding the non-randomised study, ${ }^{6}$ whereby the statistical results did not change (OR 14.51; 95\% CI 3.06 to $68.85 ; p=0.0008$ ). Owing to the poor definition and heterogeneity $\left(\mathrm{I}^{2}=73.9 \%\right)$ of criteria for the intraoperative bleeding, the result should be interpreted with caution.

Four studies reported data for the frequency of endodiathermy. ${ }^{3-5} 9$ Analysis of these data showed that the frequency of endodiathermy was statistically significantly less in the treatment group than in the control group (OR 15.06; 95\% CI 4.01 to 56.53; $p<0.0001$ ) (figure $1 B$ ).

Three studies reported data for the number of iatrogenic retinal tears. ${ }^{359}$ Analysis of these data showed that the incidence of iatrogenic retinal tears was almost significantly less in the IVB treatment group (OR 3.72; 95\% CI 1.00 to 13.79; $\mathrm{p}=0.05$ ) (figure $1 \mathrm{C}$ ).

Five studies reported data for the mean surgical time. ${ }^{1} 3469$ Analysis of these data showed that the mean surgical time was statistically significantly shorter in the treatment group than in the control group (WMD 14.13; 95\% CI 1.17 to 27.09; $p=0.03$ ) (figure 1D). A sensitivity analysis was performed to examine the effect of excluding the non-randomised study, ${ }^{6}$ and the statistical results did not change (WMD 21.59; 95\% CI 15.89 to 27.30; $\mathrm{p}<0.00001)$. This comparison generated a significant heterogeneity $\left(\mathrm{I}^{2}=75.9 \%\right)$, and the result must be interpreted with caution.

\section{Postoperative outcome parameters}

Two studies reported data for the reabsorption time of blood after surgery. ${ }^{16}$ Analysis of these data showed that the mean reabsorption time was statistically significantly less in the IVB pretreatment group than in the vitrectomy alone group (WMD 15.82; $95 \%$ CI 0.43 to $31.20 ; \mathrm{p}=0.04$ ) (figure $2 \mathrm{~A}$ ). A sensitivity analysis was performed to examine the effect of excluding the non-randomised study, ${ }^{6}$ and the statistical results did not change (WMD 23.70; 95\% CI 18.01 to 29.39; $\mathrm{p}<0.00001$ ).

Five studies reported data for the proportion of recurrent VH. ${ }^{14-69}$ The studies used differential scales for recurrent $\mathrm{VH}$ evaluation. Clinical significant recurrent $\mathrm{VH}$ (severe recurrent $\mathrm{VH}$ with no fundus details) were included in this meta-analysis. Furthermore, all incidences of recurrent $\mathrm{VH}$ during follow-ups were included. Analysis of these data showed that the proportion of recurrent $\mathrm{VH}$ was almost statistically significantly less in the treatment group than in the control group (OR 5.48; $95 \%$ CI 0.97 to $31.02 ; \mathrm{p}=0.05$ ) (figure $2 \mathrm{~B}$ ). A sensitivity analysis was performed to examine the effect of excluding the non-randomised study, ${ }^{6}$ whereby the statistical results did not change (OR 7.52; $95 \%$ CI 0.97 to $58.40 ; \mathrm{p}=0.05$ ).

Owing to the poor definition and heterogeneity $\left(\mathrm{I}^{2}=93.6 \%\right.$, $73.2 \%$, respectively) of criteria for the reabsorption time and recurrent $\mathrm{VH}$, the above two results should be interpreted with caution.

Six studies reported data for BCVA. ${ }^{13-69}$ Analysis of these data showed that the mean BCVA was statistically significantly better in the treatment group than in the control group (WMD: 0.31 ; $95 \%$ CI 0.10 to 0.52 ; $\mathrm{p}=0.003$ ) (figure $2 \mathrm{C}$ ).

Six studies reported data for postoperative complications. ${ }^{13-69}$ Analysis of these data showed no statistically significant differences in the early ( $<1$ week) elevation of IOP ( $\geq 25 \mathrm{~mm} \mathrm{Hg}$ ), late ( $\geq 1$ week) elevation of IOP ( $\geq 25 \mathrm{~mm} \mathrm{Hg}$ ), incidence of final RD and proportion of repeat vitrectomy (table 3 ). 
Comparison: Intraoperative bleeding

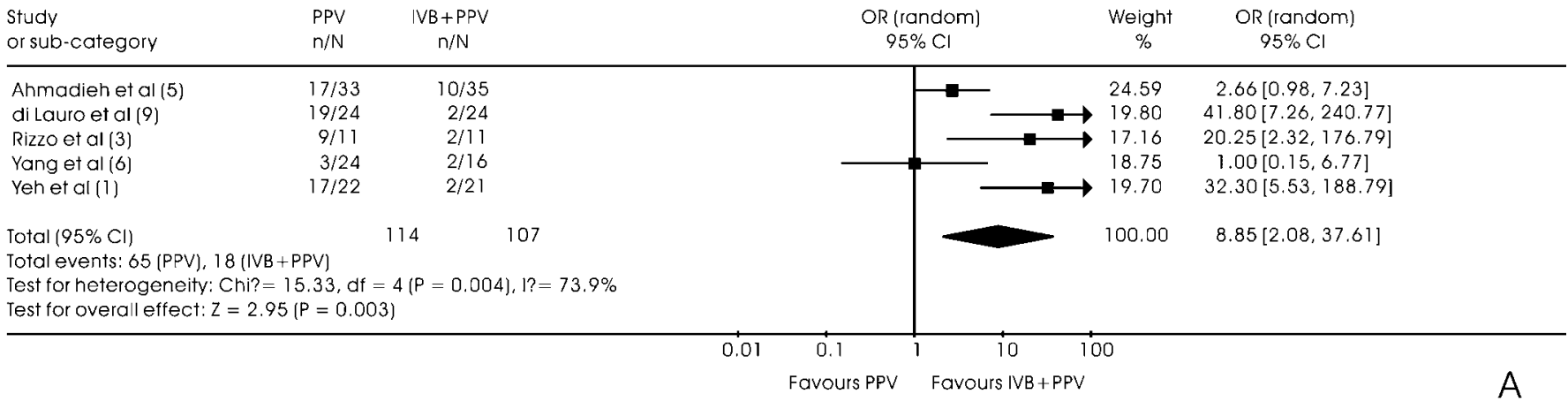

Comparison: Frequency of endodiathermy

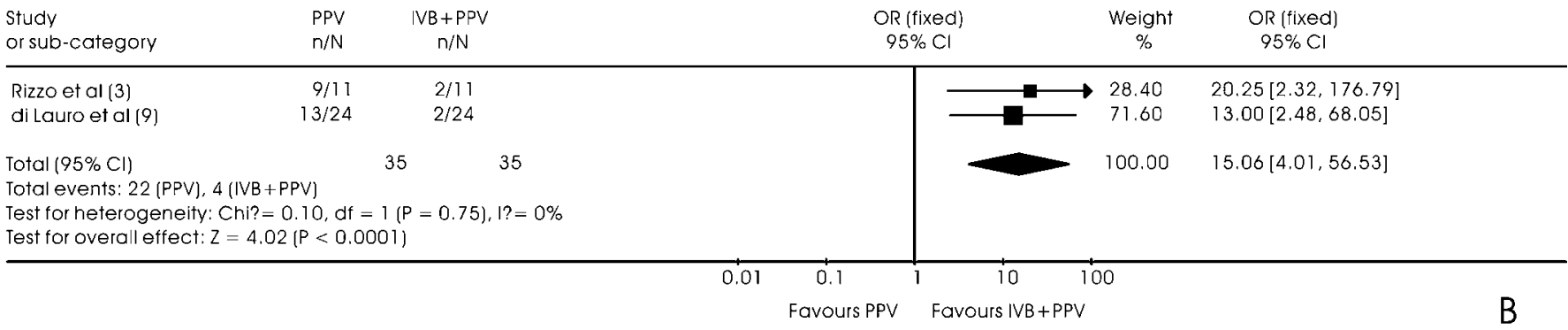

Comparison: latrogenic retinal tears

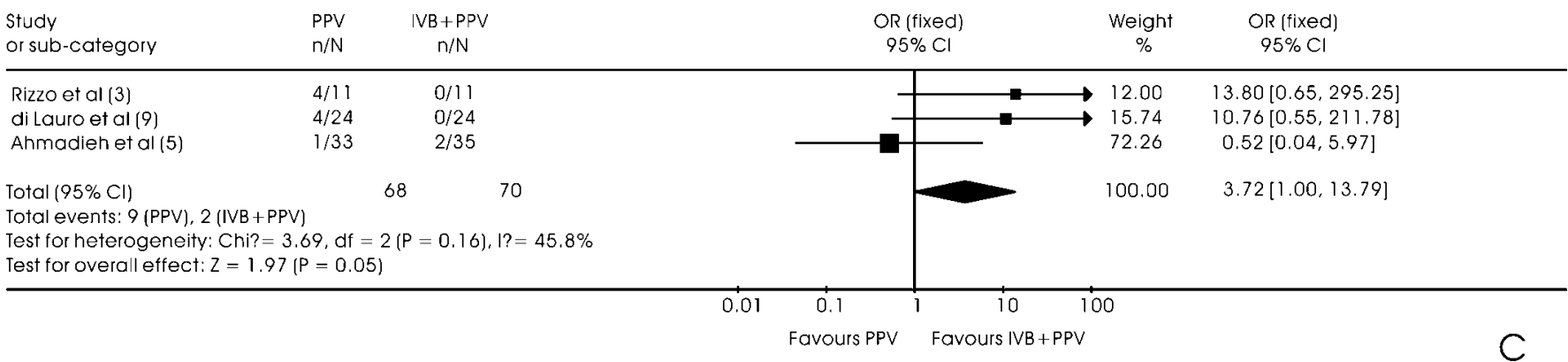

Comparison: Mean surgical time

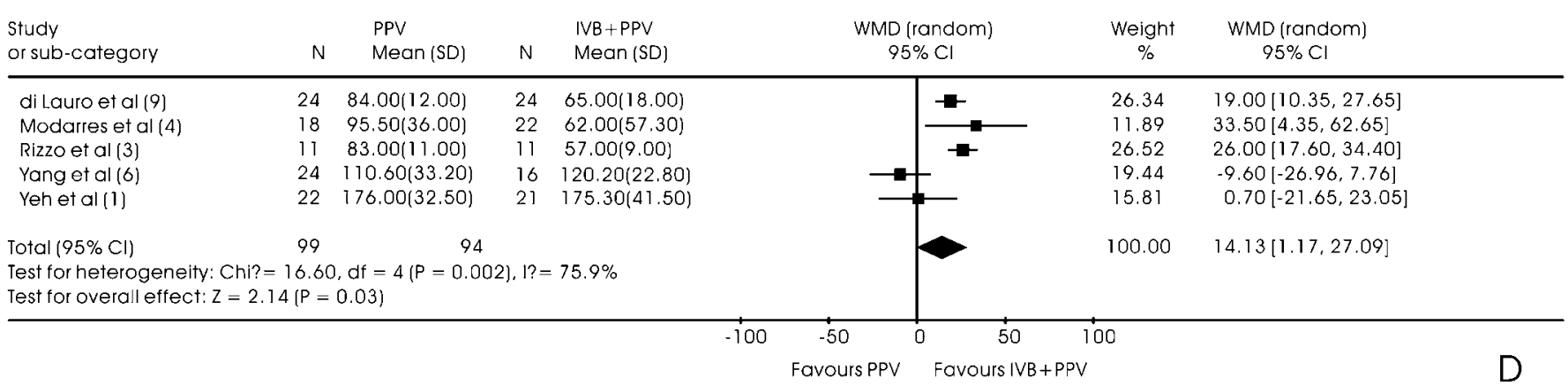

Figure 1 Forest plots of $O R$ of proportions of intraoperative bleeding $(A)$, frequency of endodiathermy $(B)$, iatrogenic retinal tears $(C)$ during vitrectomy and forest plot of WMD of mean surgical time (D) comparing vitrectomy alone to vitrectomy with intravitreal bevacizumab (IVB) pretreatment. PPV, pars plana vitrectomy; WMD, weighted mean difference.

\section{Publication bias}

Based on a visual analysis of the funnel plots, no obvious evidence of publication bias was found (figure 3).

\section{DISCUSSION}

The present meta-analysis revealed that IVB injection before vitrectomy for PDR can reduce intraoperative bleeding and the frequency of endodiathermy, and shorten the mean surgical time. It can also shorten reabsorption time of blood after vitrectomy, decrease the incidence of recurrent $\mathrm{VH}$ and improve BCVA.

Bevacizumab is a recombinant humanised monoclonal antivascular endothelial growth factor antibody used to induce regression of neovascularisation and reduce permeability. ${ }^{14}$ It has increasingly been used to treat choroidal neovascularisation and 
Comparison: Reabsorption time of blood

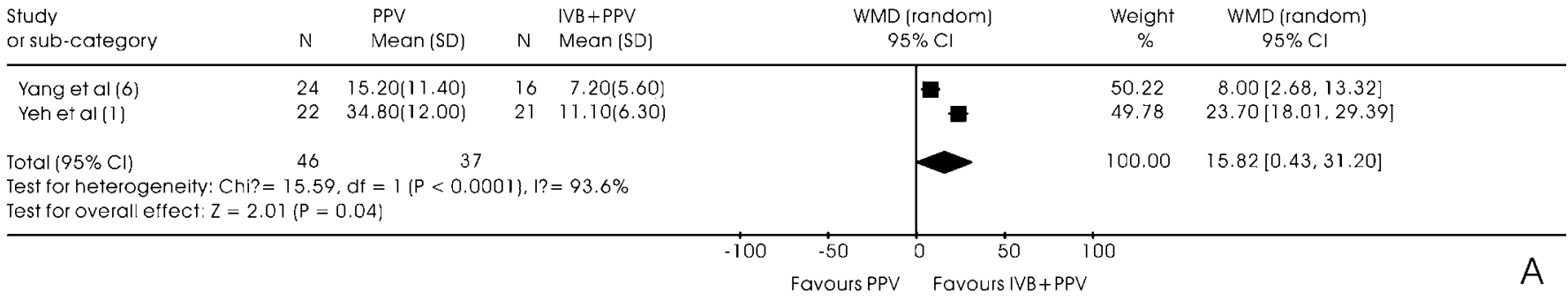

Comparison: RecurrentVH

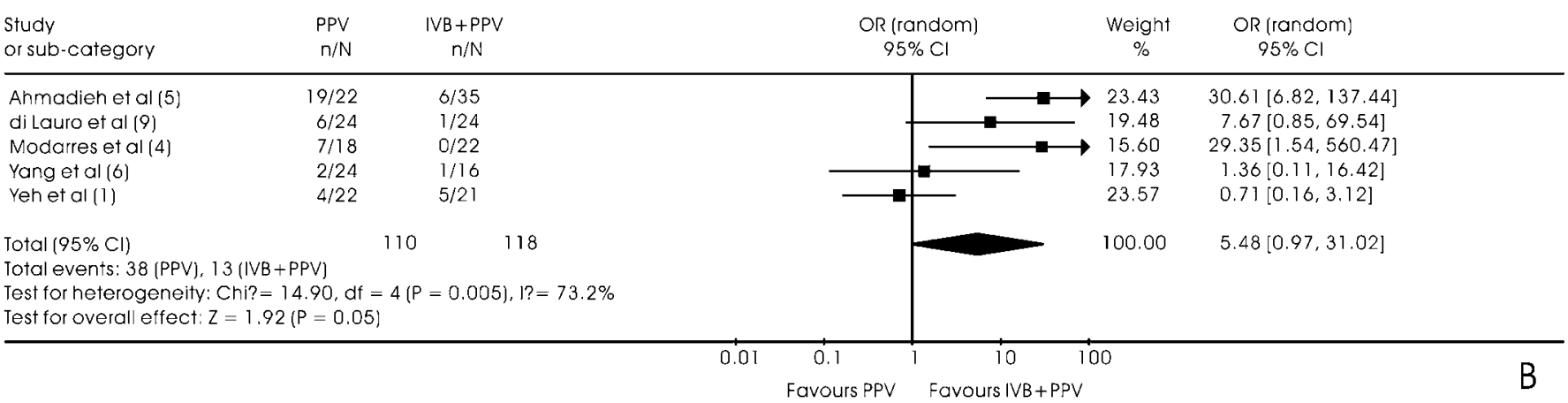

Comparison: Postoperative BCVA

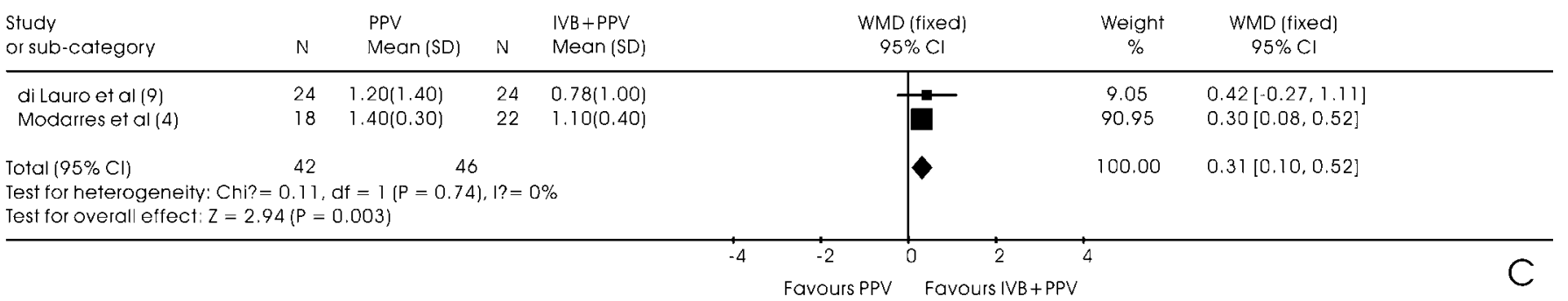

Figure 2 Forest plots of weighted mean difference (WMD) of reabsorption time of blood (A), OR of proportions of recurrent vitreous haemorrhage (B) and WMD of final mean BCVA (C) after surgery comparing vitrectomy alone to vitrectomy with intravitreal bevacizumab (IVB) pretreatment. PPV, pars plana vitrectomy.

diabetic macular oedema. ${ }^{15-18}$ It has also proven to be effective for the treatment of PDR complicated by $\mathrm{VH} .{ }^{19-21}$ Results of fluorescein angiography revealed a reduction in leakage from the foci of neovascularisation and regression of the neovascular component of fibrovascular tissue in eyes with PDR within 1 week after IVB.

Based on these observations, it was suggested that IVB may reduce the incidence of intraoperative and postoperative haemorrhage in diabetic vitrectomy. ${ }^{22}$ Chen first reported that preoperative IVB was helpful in facilitating vitrectomy in severe PDR. $^{23}$ Many clinical trials proved that IVB pretreatment ameliorates fundus conditions before vitrectomy. In Ahmadieh's study, owing to a significant resolution of $\mathrm{VH}$ and improvement of vision after IVB injection, nine eyes (25.7\%) initially scheduled for vitrectomy obviated the need for surgical intervention. ${ }^{5}$ Laboratory experiment provided objective, quantifiable data that a lower number of erythrocytes retrieved from the vitrectomy cassette were observed in those patients treated with IVB 2 weeks before surgery. ${ }^{2}$

From surgeons' experiences, the regression of the vascular component of the fibrovascular complexes after IVB facilitates segmentation and delamination of membranes. ${ }^{1}$ This is because they are less adhesive to the underlying retina and readily separated from the retina. The haemodynamic changes in retinal

Table 3 Postoperative complications of vitrectomy alone versus vitrectomy with intravitreal bevacizumab pretreatment in the meta-analysis

\begin{tabular}{|c|c|c|c|c|c|}
\hline \multirow[b]{2}{*}{ Complications } & \multirow[b]{2}{*}{ No of studies } & \multicolumn{2}{|c|}{ Crude rate, n/N (\%) } & \multirow[b]{2}{*}{$\begin{array}{l}\text { Percentage rate } \\
\text { difference }(95 \% \mathrm{CI})\end{array}$} & \multirow[b]{2}{*}{$\begin{array}{l}\text { p Value for } \\
\text { overall effect }\end{array}$} \\
\hline & & $\begin{array}{l}\text { Pars plana } \\
\text { vitrectomy group }\end{array}$ & $\begin{array}{l}\text { Intravitreal bevacizumab+pars } \\
\text { plana vitrectomy group }\end{array}$ & & \\
\hline $\begin{array}{l}\text { Early elevation of intraocular pressure } \\
(\geq 25 \mathrm{~mm} \mathrm{Hg} \text { ( } \leq 1 \text { week) }\end{array}$ & $3^{135}$ & $13 / 66$ & $12 / 67$ & $1.08(0.36$ to 3.27$)$ & 0.89 \\
\hline $\begin{array}{l}\text { Late elevation of intraocular pressure } \\
(\geq 25 \mathrm{~mm} \mathrm{Hg}) \text { ( }>1 \text { week) }\end{array}$ & $3^{136}$ & $8 / 57$ & $0 / 48$ & $17.00(0.91$ to 319.22$)$ & 0.06 \\
\hline Final retinal detachment & $3^{134}$ & $5 / 51$ & $2 / 54$ & $2.44(0.52$ to 11.46$)$ & 0.26 \\
\hline Repeat vitrectomy & $2^{49}$ & $3 / 42$ & $1 / 46$ & $2.69(0.37$ to 19.33$)$ & 0.32 \\
\hline
\end{tabular}




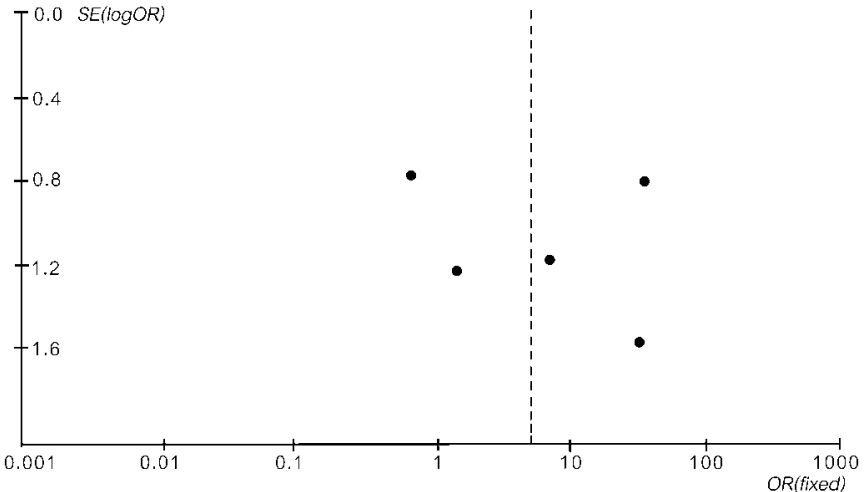

Figure 3 Funnel plot of randomised controlled trials.

circulation, such as constriction and a decreased flow in new vessels, greatly reduce the likelihood of intraoperative bleeding. Besides, decreased bleeding makes frequent adjustments of the infusion bottle height unnecessary. Fluctuations in intraocular pressure, which can damage the vulnerable disc, are thus avoided. The resolution of $\mathrm{VH}$ and less intraoperative bleeding may in turn provide a clear surgical field facilitating surgery.

Some studies reported other indexes on surgical manoeuvres, such as the frequency of required endodiathermy, and blunt and sharp membrane dissection. The surgical complexity of the removal of fibrovascular membranes directly correlates with the pathophysiology of PDR, type of vitreoretinal adhesion, and degree of the intraoperative bleeding. Rizzo's experiences provided a more direct impression. ${ }^{3}$ For bevacizumab-pretreated cases, simple blunt dissection or direct membrane peeling can be more frequent. The regression and subsequent fibrosis of retinal $\mathrm{NV}$ after IVB made the surgery quicker, requiring fewer tool exchanges, thus shortening surgical time.

Two studies reported that iatrogenic retinal breaks occurred more in the vitrectomy alone group. ${ }^{3} 9$ It was presumed that after IVB, vascular leakage, as well as the retinal thickness and congestion, were reduced, and the tissue was more resistant to traction. Less intraoperative bleeding may also provide a clear retinal visualisation, which in turn causes fewer iatrogenic breaks. Although the present meta-analysis does not fully substantiate this presumption, future larger sample size comparative clinical trials may prove it.

The rate of recurrent $\mathrm{VH}$ is an important factor evaluating whether the IVB pretreatment could achieve better clinical outcomes. The meta-analysis for all incidences of recurrent $\mathrm{VH}$ during follow-ups revealed that the IVB pretreatment reduced the rate of recurrent $\mathrm{VH}$. The meta-analysis, including the early incidences of recurrent $\mathrm{VH}$ ( $\leq 1$ week), did not change the results (omission of statistical process). This is possible, owing to minimal surgical trauma. The consequential effect of IVB ought to be considered also. Previous research revealed that bevacizumab can be detected in the retinal tissue 14 days after intravitreal injection. ${ }^{24} 25$ Although a great extent of the bevacizumab in the vitreous body stemming from the pretreatment is possibly removed during vitrectomy, the remainder in the retina may still have an impact. This local factor is partly attributed to the shortened, postsurgical vitreous clear-up time. Other factors related to the clear-up speed of the vitreous after vitrectomy for PDR include the amount of residual blood at the end of the operation, bleeding from surgically injured retinal blood vessels, bleeding from unreleased fibrovascular tissues, fibrin and inflammatory cells within the vitreous. Evidently, IVB pretreatment could reduce these factors.
Facilitating early visual rehabilitation for patients is another important clinical outcome. This positive effect may be due to minimal injury to the retinal tissue during surgery, a minimal extent of haemorrhage and posterior focal reproliferation, or clear visual pathway after surgery in bevacizumab-treated cases. ${ }^{1}$ The studies included did not report apparent macular oedema in patients with diabetes before and after vitrectomy, but surgical manoeuvre or surgical trauma, such as delamination, could influence macular physiology function. The effect of reducing diabetic macular oedema might play a role. Many clinical trials have shown that IVB can reduce diabetic macular oedema and result in a better visual recovery. ${ }^{26} 27$

Di Lauro compared a 7-day with 20-day previtrectomy administration of bevacizumab. ${ }^{9}$ Clinical outcomes showed no statistically significant differences between the two groups, but intraoperative severe bleeding, frequency of endodiathermy, iatrogenic retinal break, silicone-oil tamponade, surgical mean time and the rate of recurrent $\mathrm{VH}$ featured more in the 20-day group. Concerns about potential harm of increasing vitreoretinal traction owing to rapid neovascular involution with fibrosis contraction by IVB pretreatment must be raised. Many studies have noted complications of aggravation of fibrosis after IVB injection for active progressive PDR. ${ }^{20} 23$ One recent study found that tractional retinal detachments were worsened or newly created in 11 eyes $(5.2 \%)$ of 211 IVB injections in severe PDR. ${ }^{28}$ The time interval between IVB injection and diagnosis of tractional retinal detachment was on average 13 days. ${ }^{28}$ Ishikawa performed IVB injections in eight eyes and found that two eyes, injected 7 days before the operation, had severe fibrosis, resulting in some surgical complications. ${ }^{22}$ The evidence available indicates that the effect of IVB on regression of new vessels is rapid, often evident after $24 \mathrm{~h} .{ }^{29}$ It seems that 3-5 days or even less time is adequate for IVB to exert its antiangiogenic effect. A dosage of $1.25 \mathrm{mg}$ is commonly used. Some studies have revealed that IVB at doses of 1.25 to $2.5 \mathrm{mg}$ provide similar clinical outcomes in diabetic retinopathy. ${ }^{30} 31$ Clarification of optimal interval time and dosage of IVB still needs an RCT with a larger sample size.

The selected trials revealed that no local and systemic complications related to IVB were observed in the treatment groups. The postoperative final $\mathrm{RD}$ and repeat surgery showed no significant differences. These are possibly attributable to the natural process of PDR. Long-term follow-up studies (>6 months) are needed.

This meta-analysis may have some limitations. First, we cannot fully exclude publication bias. The number of included studies is insufficient to carry out a further statistical test, to detect publication bias through asymmetry plot. In addition, we did not attempt to gain access to unpublished results. Second, the studies included were heterogeneous in terms of study location, population, number of patients from different studies and basal condition. Access to individual-level data could certainly have improved the quality of adjustment as well as the precision of estimates. Third, there was a large disparity in study quality (relative methodological strengths and weaknesses), as reflected by the Jadad score. This review unfortunately did not comprise a sufficient number of studies of a high level Jadad score to justify a performance of subgroup metaanalyses.

In conclusion, preoperative IVB may represent a new strategy to make vitrectomy safer and more effective for severe PDR. RCTs with larger sample sizes or systematic studies are needed to better evaluate the long-term benefits and safety of bevacizumab pretreatment for severe PDR. 
Funding This work has been supported by two grants: (1) Shanghai Leading Academic Discipline Project (Project Number: S30205); (2) General Program of Bio-medical Division of Shanghai Science and Technology Commission (Project Number: 10411966200)

Competing interests None.

Provenance and peer review Not commissioned; externally peer reviewed.

\section{REFERENCES}

1. Yeh PT, Yang CM, Lin YC, et al. Bevacizumab pretreatment in vitrectomy with silicone oil for severe diabetic retinopathy. Retina 2009;29:768-74.

2. da R Lucena D, Ribeiro JA, Costa RA, et al. Intraoperative bleeding during vitrectomy for diabetic tractional retinal detachment with versus without preoperative intravitreal bevacizumab (IBeTra study). Br J Ophthalmol 2009:93:688-91.

3. Rizzo S, Genovesi-Ebert F, Di Bartolo E, et al. Injection of intravitreal bevacizumab (Avastin) as a preoperative adjunct before vitrectomy surgery in the treatment of severe proliferative diabetic retinopathy (PDR). Graefes Arch Clin Exp Ophthalmol 2008;246:837-42

4. Modarres M, Nazari H, Falavariani KG, et al. Intravitreal injection of bevacizumab before vitrectomy for proliferative diabetic retinopathy. Eur J Ophthalmol 2009;19:848-52.

5. Ahmadieh H, Shoeibi N, Entezari M, et al. Intravitreal bevacizumab for prevention of early postvitrectomy hemorrhage in diabetic patients: a randomized clinical trial. Ophthalmology 2009;116:1943-8.

6. Yang $\mathbf{C M}$, Yeh PT, Yang $\mathrm{CH}$, et al. Bevacizumab pretreatment and long-acting gas infusion on vitreous clear-up after diabetic vitrectomy. Am J Ophthalmol 2008;146:211-17.

7. Lo WR, Kim SJ, Aaberg TM Sr, et al. Visual outcomes and incidence of recurrent vitreous hemorrhage after vitrectomy in diabetic eyes pretreated with bevacizumab (avastin). Retina 2009:29:926-31.

8. Yeoh J, Williams $\mathrm{C}$, Allen $\mathrm{P}$, et al. Avastin as an adjunct to vitrectomy in the management of severe proliferative diabetic retinopathy: a prospective case series. Clin Exp Ophthalmol 2008:36:449-54.

9. di Lauro R, De Ruggiero P, di Lauro R, et al. Intravitreal bevacizumab for surgical treatment of severe proliferative diabetic retinopathy. Graefes Arch Clin Exp Ophthalmol 2010;248:785-91.

10. Romano MR, Gibran SK, Marticorena J, et al. Can an intraoperative bevacizumab injection prevent recurrent postvitrectomy diabetic vitreous hemorrhage? Eur $\mathrm{J}$ Ophthalmol 2009;19:618-21.

11. Pogue J, Yusuf S. Overcoming the limitations of current meta-analysis of randomized controlled trials. Lancet 1998:351:47-52.

12. Moher D, Cook DJ, Eastwood S, et al. Improving the quality of reports of metaanalyses of randomized controlled trials: the QUOROM statement. Quality of Reporting of Meta-analyses. Lancet 1999;354:1896-900.

13. O'Connor D, Green S, Higgins JP. Defining the review question and developing criteria for including studies. Section 5.7: Changing review questions. In: Higgins JP, Green S, eds. Cochrane Handbook for Systematic Reviews of Interventions. Version 5.0.1. Part 2: General Methods for Cochran Reviews. Oxford: Cochrane Collaboration; http://www.cochrane-handbook.org/. (accessed 20 Nov 2008).

14. Gunther JB, Altaweel MM. Bevacizumab (Avastin) for the treatment of ocular disease. Surv Ophthalmol 2009;54:372-400.
15. Soheilian M, Ramezani A, Obudi A, et al. Randomized trial of intravitreal bevacizumab alone or combined with triamcinolone versus macular photocoagulation in diabetic macular edema. Ophthalmology 2009;116:1142-50.

16. Parravano M, Menchini F, Virgili G. Antiangiogenic therapy with anti-vascular endothelial growth factor modalities for diabetic macular oedema. Cochrane Database Syst Rev 2009:(4):CD007419.

17. Weigert G, Michels S, Sacu S, et al. Intravitreal bevacizumab (Avastin) therapy versus photodynamic therapy plus intravitreal triamcinolone for neovascular age-related macular degeneration: 6-month results of a prospective, randomised, controlled clinical study. Br J Ophthalmol 2008;92:356-60.

18. Ip MS, Scott IU, Brown GC, et al; American Academy of Ophthalmology. Antivascular endothelial growth factor pharmacotherapy for age-related macular degeneration: a report by the American Academy of Ophthalmology. Ophthalmology 2008;115:1837-46

19. Avery RL, Pearlman J, Pieramici DJ, et al. Intravitreal bevacizumab (Avastin) in the treatment of proliferative diabetic retinopathy. Ophthalmology 2006;113:1695-705. e1-15.

20. Moradian S, Ahmadieh $\mathrm{H}$, Malihi $\mathrm{M}$, et al. Intravitreal bevacizumab in active progressive proliferative diabetic retinopathy. Graefes Arch Clin Exp Ophthalmol 2008:246:1699-705

21. Jorge R, Costa RA, Calucci D, et al. Intravitreal bevacizumab (Avastin) for persistent new vessels in diabetic retinopathy (IBEPE study). Retina 2006:26:1006-13.

22. Ishikawa K, Honda S, Tsukahara Y, et al. Preferable use of intravitreal bevacizumab as a pretreatment of vitrectomy for severe proliferative diabetic retinopathy. Eye (Lond) 2009:23:108-11.

23. Chen $\mathbf{E}$, Park $\mathrm{CH}$. Use of intravitreal bevacizumab as a preoperative adjunct for tractional retinal detachment repair in severe proliferative diabetic retinopathy. Retina 2006;26:699-700.

24. Bakri SJ, Snyder MR, Reid JM, et al. Pharmacokinetics of intravitreal bevacizumab (Avastin). Ophthalmology 2007:114:855-9.

25. Heiduschka P, Fietz $\mathrm{H}$, Hofmeister $\mathrm{S}$, et al. Penetration of bevacizumab through the retina after intravitreal injection in the monkey. Invest Ophthalmol Vis Sci 2007:48:2814-23.

26. Ahmadieh H, Ramezani A, Shoeibi N, et al. Intravitreal bevacizumab with or withou triamcinolone for refractory diabetic macular edema; a placebo-controlled, randomized clinical trial. Graefes Arch Clin Exp Ophthalmol 2008;246:483-9.

27. Scott IU, Edwards AR, Beck RW, et al; Diabetic Retinopathy Clinical Research Network. A phase II randomized clinical trial of intravitreal bevacizumab for diabetic macular edema. Ophthalmology 2007;114:1860-7.

28. Arevalo JF, Maia M, Flynn HW Jr, et al. Tractional retinal detachment following intravitreal bevacizumab (Avastin) in patients with severe proliferative diabetic retinopathy. Br J Ophthalmol 2008;92:213-16.

29. Spaide RF, Fisher YL. Intravitreal bevacizumab treatment of proliferative diabetic retinopathy complicated by vitreous hemorrhage. Retina 2006:26:275-8.

30. Lam DS, Lai TY, Lee VY, et al. Efficacy of $1.25 \mathrm{MG}$ versus $2.5 \mathrm{MG}$ intravitreal bevacizumab for diabetic macular edema: six-month results of a randomized controlled trial. Retina 2009;29:292-9.

31. Arevalo JF, Sanchez JG, Fromow-Guerra J, et al. Comparison of two doses of primary intravitreal bevacizumab (Avastin) for diffuse diabetic macular edema: results from the Pan-American Collaborative Retina Study Group (PACORES) at 12-month follow-up. Graefes Arch Clin Exp Ophthalmol 2009;247:735-43.

Online Archive

Visit our Online Archive - available back to 1917. Subscribers may access the entire archive freely. Non-subscribers have free access to all articles prior to 2006. A simple one-time registration is required that grants access to all the free archive content, across all of our specialist titles. To view or to register visit bjo.bmj.com. 